\title{
First-Principles Study of Vacancy and Impurities Defects in Graphene
}

\author{
Hari Krishna Neupane ${ }^{1,2}$, Narayan Prasad Adhikari ${ }^{2 *}$ \\ ${ }^{1}$ Amrit Campus, Institute of Science and Technology Tribhuvan University, Kathmandu, Nepal \\ ${ }^{2}$ Central Department of Physics, Institute of Science and Technology Tribhuvan University, Kathmandu, Nepal \\ *E-mail: narayan.adhikari@cdp.tu.edu.np
}

(Received:29 January 2021, Received in revised form: 18 April, Accepted: 25 June 2021, Available Online)

\section{Highlights}

- $\quad$ Electronic and magnetic properties of vacancy and impurity defects in graphene are studied by using first-principles calculations through computational tool Quantum ESPRESSO.

- $\quad$ From band and DOS calculations, all the defected materials are found to be metallic.

- By the analysis of DOS and PDOS calculations, $1 \mathrm{C}$ vacancy and $1 \mathrm{~N}$ impurity defects in graphene have magnetic properties, but 10 mpurity defect in graphene has non-magnetic properties.

\begin{abstract}
In this work, we have studied the electronic and magnetic properties of $1 C$ atom vacancy defects in graphene (1C $\left.{ }_{v-d}-G\right)$, $1 N$ atom impurity defects in graphene $\left(1 N_{I-d}-G\right)$ and 10 atom impurity defects in graphene $\left(1 O_{I-d}-G\right)$ materials through firstprinciples calculations based on spin-polarized density functional theory (DFT) method, using computational tool Quantum ESPRESSO (QE) code. From band structure and density of states (DOS) calculations, we found that supercell structure of monolayer graphene is a zero bandgap material. But, electronic bands of $1 C_{v-d}-G, 1 N_{I-d}-G$ and $1 O_{I-d}-G$ materials split around the Fermi energy level and DOS of up \& down spins states appear in the Fermi energy level. Thus, $1 C_{v-d}-G, 1 N_{I-d}-G$ and $1 O_{I-}$ ${ }_{d}-G$ materials have metallic properties. We have studied the magnetic properties of pure and defected materials by analyzing density of states (DOS) and partial density of states (PDOS) calculations. We found that graphene and $1 O_{I-d}-G$ materials have non-magnetic properties. On the other hand, $1 C$ vacancy atom and $1 N$ impurity atom induced magnetization in $1 C_{v-d}-G \& 1 N_{I-}$ ${ }_{d}-G$ materials by the rebonding of dangling bonds and acquiring significant magnetic moments of values $-0.75 \mu_{B} /$ cell \& $0.05 \mu_{B}$ cell respectively through remaining unsaturated dangling bond. Therefore, non-magnetic graphene changes to magnetic $1 C_{v-d}-G$ and $1 N_{I-d}-G$ materials due to $1 C$ atom vacancy defects and $1 N$ atom impurity defects. The $2 p$ orbital of carbon atoms has main contribution of magnetic moment in these defected structures.
\end{abstract}

Keywords: DFT; vacancy defects; impurity defects; graphene; magnetic moment

\section{Introduction}

Graphene is a two dimensional (2D) stretchable, $\mathrm{sp}^{2}$ hybridized single sheet of carbon atoms organized in a honeycomb lattice structure. Its hexagonal structure is stable in which each carbon atom bonds with the surrounding carbon atoms and non-bonding electrons move freely between the layers. Dirac cones provided by the linearly crossing $\pi$ and $\pi^{*}$ bands meet at six points in k-space, and is called zero band gap semiconductor [1]. Graphene has series of special physical properties such as high mechanical strength, chemical stability, massless Dirac fermions behavior, ambipolar effect, unique electronic

${ }^{*}$ Corresponding author 
and magnetic properties [2-5]. It is used for the fabrication of electronic devices, transparent electrodes and spintronics devices [6-8]. Therefore, it opened up appealing opportunities for developing nanoelectronic devices. Because of growing allotropes and tunable thermal, electronic and mechanical properties through appropriate modifications, graphene has recently become a leading section of solid state physics and material science. However, the lack of intrinsic band gap and non-magnetic nature of graphene limits its practical applications in widely expanding field of carbon-based devices.

The defects in solids cause deviation of atoms or ions from the periodicity and they are used to find innovative properties. They can be used to design new materials. Among the different type of defects in solids, point defects play important role in solid-state physics. Point defect is classified in to vacancy defects and impurity defects [9-11]. The magnetic ordering in graphene is often explained by the presence of vacancies and impurities defects. Defects are expected to play key roles in the chemical functionally and electronic transport properties of graphene based materials [12-15]. So, defects in graphene provide an opportunity for the researchers. The theoretical and experimental research groups have studied the defects in monolayer structures of graphene and found that a single carbon atom vacancy defects in graphene is able to induce local magnetic moments due to the three carbon dangling bond atoms surrounding a single vacancy [16-24]. The adsorption of new atom in graphene tunes more desirable properties than pristine graphene and vacancy defects graphene. To our best knowledge, electronic and magnetic properties of graphene structure due to the impurities defects by Nitrogen $(\mathrm{N})$ atom and Oxygen $(\mathrm{O})$ atom has not been reported. Therefore, in present work, we have investigated the electronic and magnetic properties of $1 \mathrm{C}$ atom vacancy defects, $1 \mathrm{~N}$ atom and 10 atom; impurities defects in $(4 \times 4)$ supercell structure of monolayer graphene using first-principles approaches based on spin-polarized density functional theory (DFT) method.

In sections 2 and 3, we will discuss details of computational methods and interpretations of finding respectively. We closed the paper with main conclusions of the present work in section 4 .

\section{Computational details}

We used first-principles calculations based on spin-polarized density functional theory (DFT) [25], within the generalized gradient approximation (GGA) using computational tool Quantum ESPRESSO package [26, 27], and structure analysis tool XCrySDen. The exchange-correlation potential is approximated with Perdew-Burke-Ernzerhof (PBE) functional [27]. Ultra-soft pseudo-potential contained in Rappe-Rabe-Kaxiraas-Joannopoulos (RRJK) model is used to describe the chemical activity of valence electrons in calculations. At first, we have prepared optimized primitive unit cell of graphene, then we have created $(4 \times 4)$ supercell structure of monolayer graphene by extending optimized primitive unit cell along $\mathrm{x}$ and y directions. Vacancy defects structure is prepared by removing $1 \mathrm{C}$ atom from the $(4 \times 4)$ supercell structure of monolayer graphene sheet. Also, Impurities defects structures are constructed by replacing $1 \mathrm{C}$ atom by $1 \mathrm{~N}$ atom and $1 \mathrm{O}$ atom respectively in $(4 \times 4)$ supercell structure of monolayer graphene sheet. The Brillouin zone of all structures was sampled by $(6 \times 6 \times 1)$ k-points in the Monkhorst-Pack (MP) scheme [28]. A plane-wave basis set with energy cut-off value of (35 Ry) and charge density cut-off value (350 Ry) was used for the expansion of ground state electronic wave function. Atomic positions were optimized by using the Broyden-FletcherGoldfarb-Shanno (BFGS) scheme [29], until the total energy changes between two consecutive self consistent field (SCF) steps is less than $10^{-4} \mathrm{Ry}$ and each component of force acting is less than $10^{-3} \mathrm{Ry} /$ Bohrs. And, we used Marzarri-Vanderbilt (MV) [30] method of 'smearing' having width of 0.001 Ry. Spin-polarized DFT was used for the magnetic properties calculations of the systems. The meshes of $(6 \times 6 \times 1) \mathrm{k}$-points is used for electronic band structure and $(12 \times 12 \times 1) \mathrm{k}$-points is used for density of states (DOS) and partial density of states (PDOS) calculations, where $100 \mathrm{k}$-points are used along the high symmetric points connecting the reciprocal space for bands structure calculations. In present work, we have prepared $1 \mathrm{C}$ atom vacancy defects, $1 \mathrm{~N}$ atom impurity defects and $1 \mathrm{O}$ atom impurity defects in $(4 \times 4)$ supercell structure of monolayer graphene material as shown in figures 1(a-d). These impurities defected materials are formed by replacing centre position 1C atom of graphene sheet by $1 \mathrm{~N}$ atom and 10 atom respectively. The defected structures are then optimized and relaxed by BFGS method, which are used for further calculations.

\section{Results and Discussion}

In this section, we present our results for the two types of defects mentioned above and their interpretations. 


\section{Electronic properties}

The $(4 \times 4)$ supercell structure of Graphene is made by extending optimized primitive unit cell along $\mathrm{x}$ and y directions. The distance between two nearest carbon atoms in graphene is $1.417 \AA$ [21]. This value agrees with experimentally reported value $1.42 \AA[30]$. Here, we have also done the relax calculation of $(4 \times 4)$ supercell structure of graphene until the convergence is achieved, then we performed self-consistent field (SCF) calculations of supercell structure to get total energy, binding energy and binding energy per atom. The binding energy and binding energy per atom of supercell structure are calculated by using the relation;

$$
\mathrm{E}_{\mathrm{b}}=\mathrm{NE}_{\mathrm{c}}-\mathrm{E}_{\mathrm{g}}
$$

Where, $\mathrm{E}_{\mathrm{g}}$ is the ground state energy of pure graphene sheet, $\mathrm{E}_{\mathrm{c}}$ is ground state energy of isolated carbon atom and $\mathrm{N}$ is the number of carbon atom in a graphene supercell. Similarly, the binding energy per carbon atom is calculated by the relation;

$$
\mathrm{E}_{\mathrm{b} / \mathrm{C} \text {-atom }}=\left(\mathrm{NE}_{\mathrm{c}}-\mathrm{E}_{\mathrm{g}}\right) / \mathrm{N}
$$

The calculated values of total ground state energy, energy of isolated carbon atom, binding energy, and binding energy per carbon atom for graphene sheet containing $32 \mathrm{C}$ atoms (i.e. 32 carbon atoms are presented in $(4 \times 4)$ supercell structure of graphene) are -397.78 Ry, -11.84 Ry, $255.44 \mathrm{eV}$ and $7.98 \mathrm{eV} /$ atom respectively. In this work, we have investigated the electronic and magnetic properties of vacancy and impurities defects in supercell structure of graphene. The defects are localized states. They give rise to localized states in the band gap. In our work, we studied two types of defects in graphene. The 1C atom vacancy defects $\left(1 \mathrm{C}_{\mathrm{v}-\mathrm{d}}-\mathrm{G}\right)$ material is created by removing $1 \mathrm{C}$ (centre 1C) atom from $(4 \times 4)$ supercell structure of graphene as shown in figure $1(\mathrm{~b})$, where defects concentration of $1 \mathrm{C}$ atom in structure is $3.125 \%$. The $1 \mathrm{~N}$ atom impurity defects $\left(1 \mathrm{~N}_{\mathrm{s}-\mathrm{d}}-\mathrm{G}\right)$ and $1 \mathrm{O}$ atom impurity defects $\left(1 \mathrm{O}_{s-d^{-}}-\mathrm{G}\right)$ materials are prepared by replacing $1 \mathrm{C}$ atom with $1 \mathrm{~N}$ atom and $1 \mathrm{O}$ atom respectively in supercell structure of graphene as shown in figures $1(\mathrm{c}-\mathrm{d})$.
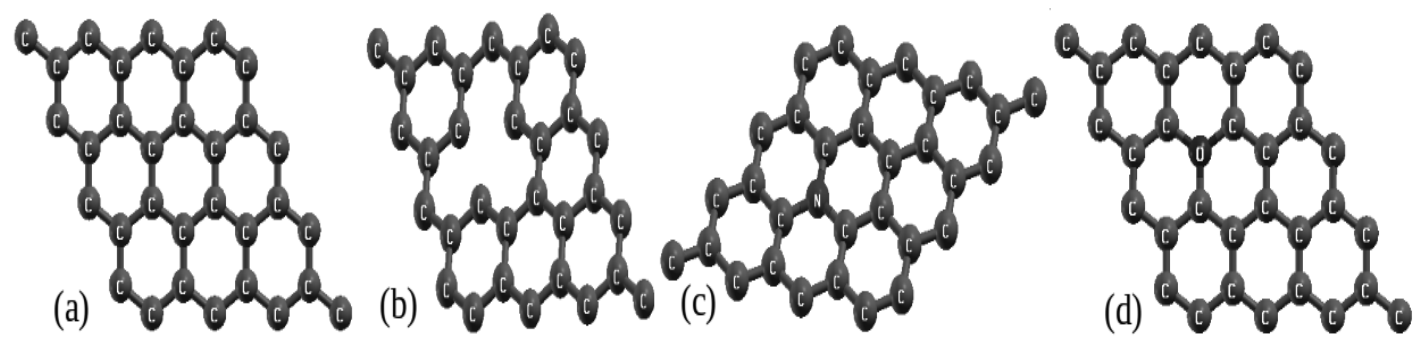

Fig. 1: Optimized and relaxed; (a) (4×4) supercell structure of graphene, (b) $1 C$ atom vacancy defects in (4 $\times 4)$ supercell structure of graphene, (c) $1 \mathrm{~N}$ atom impurity defects in $(4 \times 4)$ supercell structure of graphene, (d) 10 atom impurity defects in (4×4) supercell structure of graphene.

Electronic properties of materials are investigated by analyzing band and DOS structures calculations. Band and DOS plots of pristine graphene are shown in figures 2(a-b), where we have taken $100 \mathrm{k}$-points along the specific direction of irreducible Brillouin zone to get fine band structure by choosing $\Gamma-\mathrm{M}-\mathrm{K}-\Gamma$ high symmetric points, $\mathrm{x}$-axis represents high symmetric points in the first Brillouin zone and y-axis represents the corresponding energy value. Up \& down spins states of DOS are plotted along $y$-axis and its corresponding energy values are plotted in $x$-axis, and the vertical dotted line represents Fermi energy level and horizontal dotted line separates up \& down spins states of electrons in the orbital of all atoms present in material. We can see that Dirac point of graphene lies at the Fermi energy level in band structure and no DOS states appear at the Fermi energy level in DOS plot as shown in figures $2(a-b)$. So that pure graphene is a zero bandgap material.

In addition, $1 \mathrm{C}$ atom vacancy defects with above mentioned concentration in graphene is seen still have linearly crossing bands, and localized vacancy states corresponding to the flat impurities bands in bandgaps. Due to symmetry of the supercell having $1 \mathrm{C}$ vacancy defects, the linearly crossing bands split and they are raised slightly above the Fermi energy level, it means n-type Schottky barrier (contact) is formed. The states associated with the dangling bond and reconstructed C-C bond of vacancy occurs near the top of valence band and in the conduction band appear as flat bands and charge densities are associated these bands are localized as shown in figure 3(a). The band plots of $1 \mathrm{~N} \& 1 \mathrm{O}$ impurities defects in graphene are shown in figures 4(a) \& 5(a) respectively where, electronic bands split. As a result, they cross the Fermi energy level and appear in valence band, it means 
p-type Schottky barriers of impurities defects are formed in both structures. Thus, $\pi$ and $\pi^{*}$ bands around Fermi level mix with the orbitals of vacancy and impurities defects. In metal and semiconductor, n-type Schottky barrier means, the energy difference between Fermi energy level and conduction band minimum, and p-type Schottky barrier means energy difference between the Fermi energy level and valence band maximum. Therefore, sum of n-type and p-type Schottky barrier give energy bandgap of semiconductor $[31,32]$. Also, in DOS plots of $1 \mathrm{C}_{\mathrm{v}-\mathrm{d}}-\mathrm{G}, 1 \mathrm{~N}_{\mathrm{I}-\mathrm{d}}-\mathrm{G} \& 1 \mathrm{O}_{\mathrm{I}-\mathrm{d}}-\mathrm{G}$ materials, we observed that spins of up-DOS \& down-DOS states are appeared around the Fermi level. Therefore, from the analysis of band and DOS calculations, we found that vacancy defects material $1 \mathrm{C}_{\mathrm{v}-\mathrm{d}}-\mathrm{G}$ and impurities defects materials $1 \mathrm{~N}_{\mathrm{I}-\mathrm{d}}-\mathrm{G} \& 1 \mathrm{O}_{\mathrm{I}-\mathrm{d}}-\mathrm{G}$ have metallic nature. We know that the electronic configurations of valence electrons in $\mathrm{C}, \mathrm{N}$ and $\mathrm{O}$ atoms are [He] $2 \mathrm{~s}^{2} 2 \mathrm{p}^{2}$, [He] $2 \mathrm{~s}^{2} 2 \mathrm{p}^{3}$ and [He] $2 \mathrm{~s}^{2} 2 \mathrm{p}^{4}$ respectively. Each $\mathrm{C}$ atom has single up spin in $2 \mathrm{p}_{\mathrm{x}}, 2 \mathrm{p}_{\mathrm{y}}$ and vacant in $2 \mathrm{p}_{\mathrm{z}}$ sub-orbital, each $\mathrm{N}$ atom has single up spin in $2 \mathrm{p}_{\mathrm{x}}, 2 \mathrm{p}_{\mathrm{y}}$ and $2 \mathrm{p}_{\mathrm{z}}$ suborbitals and each $\mathrm{O}$ atom contains paired spins in $2 \mathrm{p}_{\mathrm{x}}$ sub-orbital and single unpaired up spin in $2 \mathrm{p}_{\mathrm{y}}$ and $2 \mathrm{p}_{\mathrm{z}}$ sub-orbital. Due to the arrangement of unpaired up and down spins states of electrons in the orbitals of atoms in all $1 \mathrm{C}_{\mathrm{v}-\mathrm{d}}-\mathrm{G}_{1} 1 \mathrm{~N}_{\mathrm{I}-\mathrm{d}}-\mathrm{G}^{2}$ \& $1 \mathrm{O}_{\mathrm{I}-\mathrm{d}}-\mathrm{G}_{\text {materials }}$ obtained different values of Fermi energy. We found that Fermi energy values of these materials are $-2.93 \mathrm{eV},-1.64 \mathrm{eV}$ and -1.75 $\mathrm{eV}$ respectively. Also, we calculated Fermi energy shift values of $1 \mathrm{C}_{\mathrm{v}-\mathrm{d}}-\mathrm{G}, 1 \mathrm{~N}_{\mathrm{I}-\mathrm{d}}-\mathrm{G} \& 1 \mathrm{O}_{\mathrm{I}-\mathrm{d}}-\mathrm{G}$ material from Fermi energy value of pure graphene are $0.38 \mathrm{eV},-0.91 \mathrm{eV} \&-0.80 \mathrm{eV}$ respectively as given in table 1 .

Table 1: Fermi energy $\left(\mathrm{E}_{\mathrm{f}}\right)$; Fermi energy shift from pure graphene $\left(\mathrm{E}_{\mathrm{s}}\right)$; total energy $\left(\mathrm{E}_{\mathrm{T}}\right)$; binding energy per carbon atom of graphene $(\mathrm{G})$, vacancy defects $\left(1 \mathrm{C}_{\mathrm{v}-\mathrm{d}}-\mathrm{G}\right)$, impurities defects $\left(1 \mathrm{~N}_{\mathrm{I}-\mathrm{d}}-\mathrm{G} \& 1 \mathrm{O}_{\mathrm{I}-\mathrm{d}}-\mathrm{G}\right)$ structures $\left(\mathrm{E}_{\mathrm{b}}\right)$; total value of magnetic moment (M); and magnetic moment due to total up \& down spins of electrons in $2 \mathrm{~s}, 2$ p orbitals of $\mathrm{C}, \mathrm{N} \& \mathrm{O}$ atoms $(\mu)$; in pristine graphene $(\mathrm{G}), 1 \mathrm{C}$ atom vacancy in graphene $\left(1 \mathrm{C}_{\mathrm{v}-\mathrm{d}}-\mathrm{G}\right), 1 \mathrm{~N}$ atom impurity defects in graphene $\left(1 \mathrm{~N}_{\mathrm{I}-\mathrm{d}}-\mathrm{G}\right)$ and $1 \mathrm{O}$ atom impurity defects in graphene $\left(1 \mathrm{O}_{\mathrm{I}-\mathrm{d}}-\mathrm{G}\right)$.

\begin{tabular}{|c|c|c|c|c|}
\hline $\begin{array}{l}\text { Data obtained from band, DOS \& PDOS analysis of } 1 \mathrm{C}_{\mathrm{v}-\mathrm{d}}-\mathrm{G}, 1 \mathrm{~N}_{\mathrm{I}-\mathrm{d}}-\mathrm{G} \& 1 \mathrm{O}_{\mathrm{I}-}-\mathrm{G} \\
\text { materials }\end{array}$ & $\mathrm{G}$ & $1 C_{v-d}-G$ & $1 \mathrm{~N}_{\mathrm{I}-\mathrm{d}}-\mathrm{G}$ & $1 \mathrm{O}_{\mathrm{I}-\mathrm{d}}-\mathrm{G}$ \\
\hline $\mathrm{E}_{\mathrm{f}}(\mathrm{eV})$ & -2.55 & -2.93 & -1.64 & -1.75 \\
\hline $\mathrm{E}_{\mathrm{s}}(\mathrm{eV})$ & - & 0.38 & -0.91 & -0.80 \\
\hline $\mathrm{E}_{\mathrm{T}}(\mathrm{Ry})$ & -397.78 & -352.50 & -373.46 & -403.67 \\
\hline $\mathrm{E}_{\mathrm{b}}(\mathrm{eV})$ & 7.98 & 7.46 & 7.92 & 7.97 \\
\hline$\mu$ due to $2 \mathrm{~s}$ of $\mathrm{C}$ atoms $\left(\mu_{\mathrm{B}} /\right.$ cell $)$ & 0.00 & -0.22 & 0.01 & 0.00 \\
\hline$\mu$ due to $2 p$ of $C$ atoms $\left(\mu_{\mathrm{B}} /\right.$ cell $)$ & 0.00 & -0.53 & 0.03 & 0.00 \\
\hline$\mu$ due to 2 s of $\mathrm{O}$ atom $\left(\mu_{\mathrm{B}} /\right.$ cell $)$ & - & - & - & 0.00 \\
\hline$\mu$ due to $2 p$ of $O$ atom $\left(\mu_{B} /\right.$ cell $)$ & - & - & - & 0.00 \\
\hline$\mu$ due to $2 \mathrm{~s}$ of $\mathrm{N}$ atom $\left(\mu_{\mathrm{B}} /\right.$ cell $)$ & - & - & -0.00 & - \\
\hline$\mu$ due to $2 p$ of $N$ atom $\left(\mu_{\mathrm{B}} /\right.$ cell $)$ & - & - & 0.01 & - \\
\hline Total value of magnetic moment $\mathrm{M}\left(\mu_{\mathrm{B}} /\right.$ cell $)$ & 0.00 & -0.75 & 0.05 & 0.00 \\
\hline
\end{tabular}

To understand the electronic and magnetic properties of materials more clearly, here we have carried out density of states (DOS) and partial density of states (PDOS) calculations. The DOS and PDOS plots of $1 \mathrm{C}_{\mathrm{v}-\mathrm{d}}-\mathrm{G}, 1 \mathrm{~N}_{\mathrm{I}-\mathrm{d}}-\mathrm{G}^{\mathrm{S}} 1 \mathrm{O}_{\mathrm{I}-\mathrm{d}}-\mathrm{G}_{\mathrm{materials}}$ are shown in figures 3(b-c), 4(b-c) \& 5(b-c) respectively, where vertical dotted line represents Fermi energy levels and horizontal dotted line separates up \& down spins states of electrons in the orbital of atom in present in respective structures. 


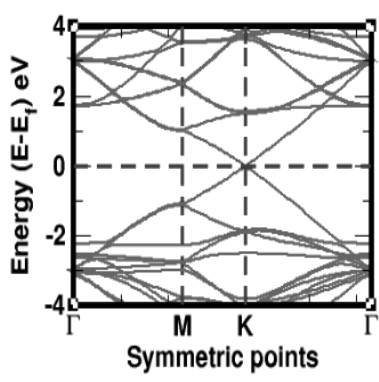

(a)

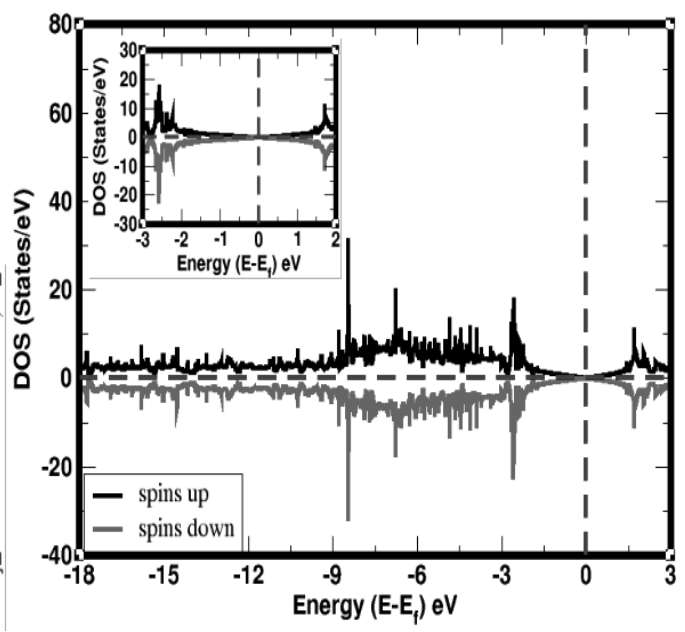

(b)

Fig. 2: (a) Band plot of $(4 \times 4)$ supercell structure of graphene, (b) DOS of up $\&$ down spins states of atoms in (4×4) supercell structure of graphene. In band structure, horizontal dotted line represents Fermi energy level, and in DOS plot, vertical dotted line represents Fermi energy level.

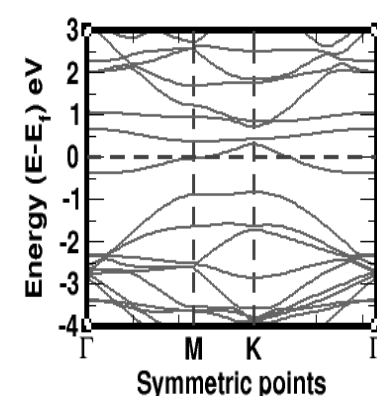

(a)

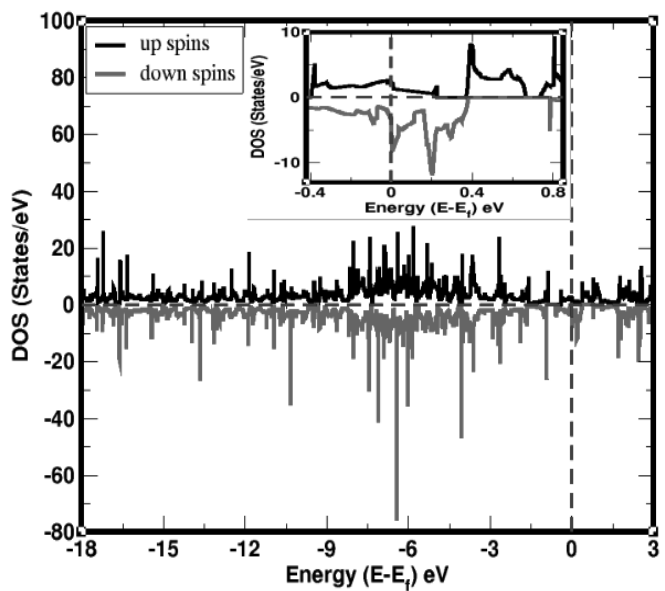

(b)

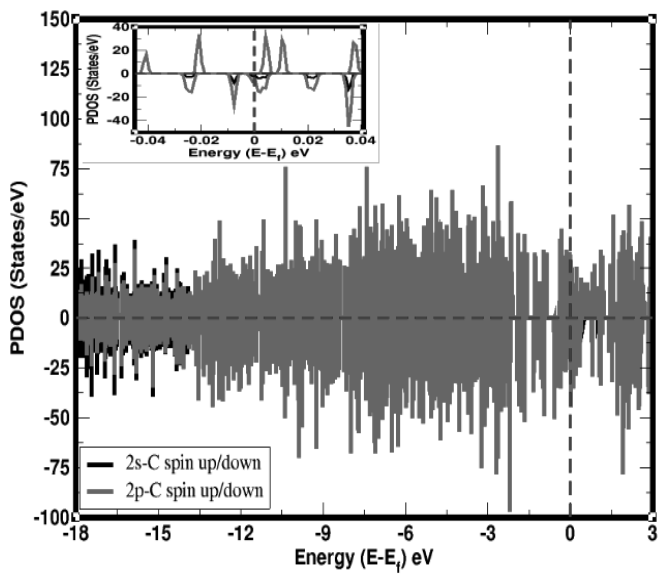

(c)

Fig. 3: (a) Band plot of $1 C$ atom vacancy defects in (4×4) supercell structure of graphene, (b) DOS of up \& down spins states of $1 C$ atom vacancy defects in (4×4) supercell structure of graphene (c) PDOS of individual up \& down spins states of orbitals of $1 C$ atom vacancy defects in (4×4) supercell structure of graphene. In band structure, horizontal dotted line represents Fermi energy level, and in DOS/PDOS plots, vertical dotted line represents Fermi energy level. 


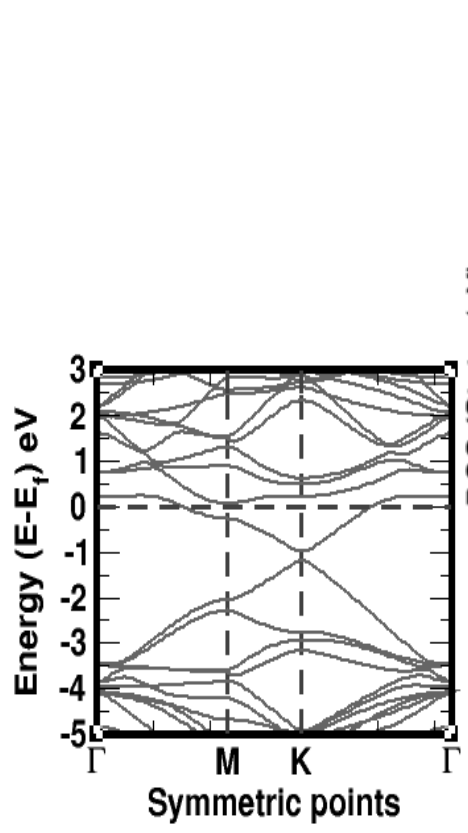

(a)

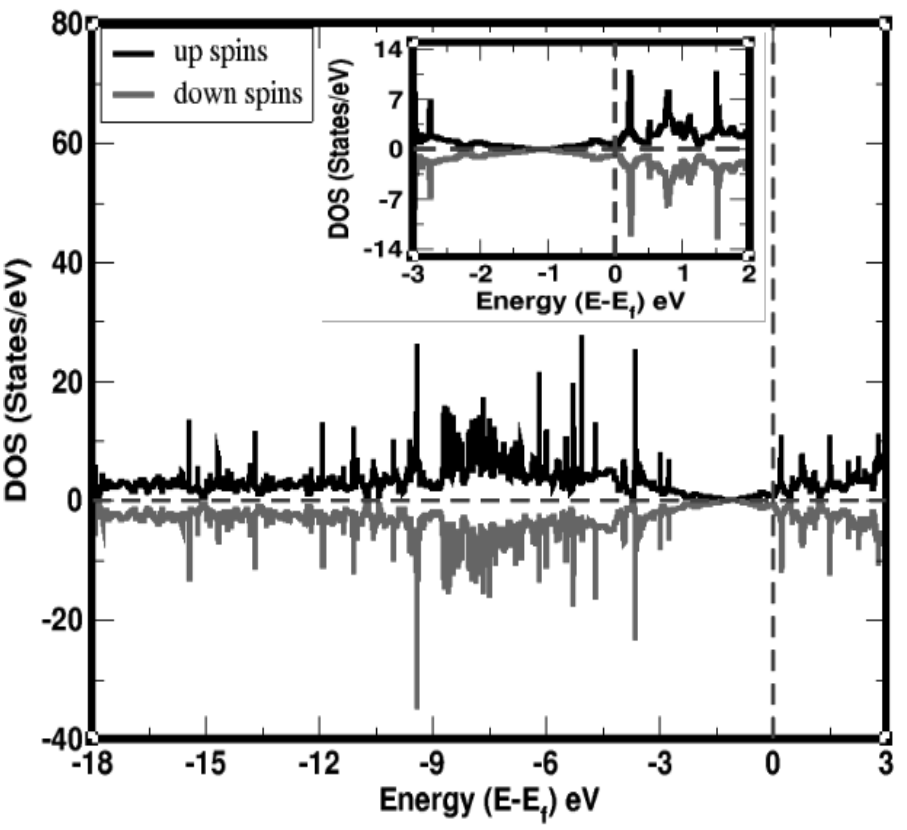

(b)

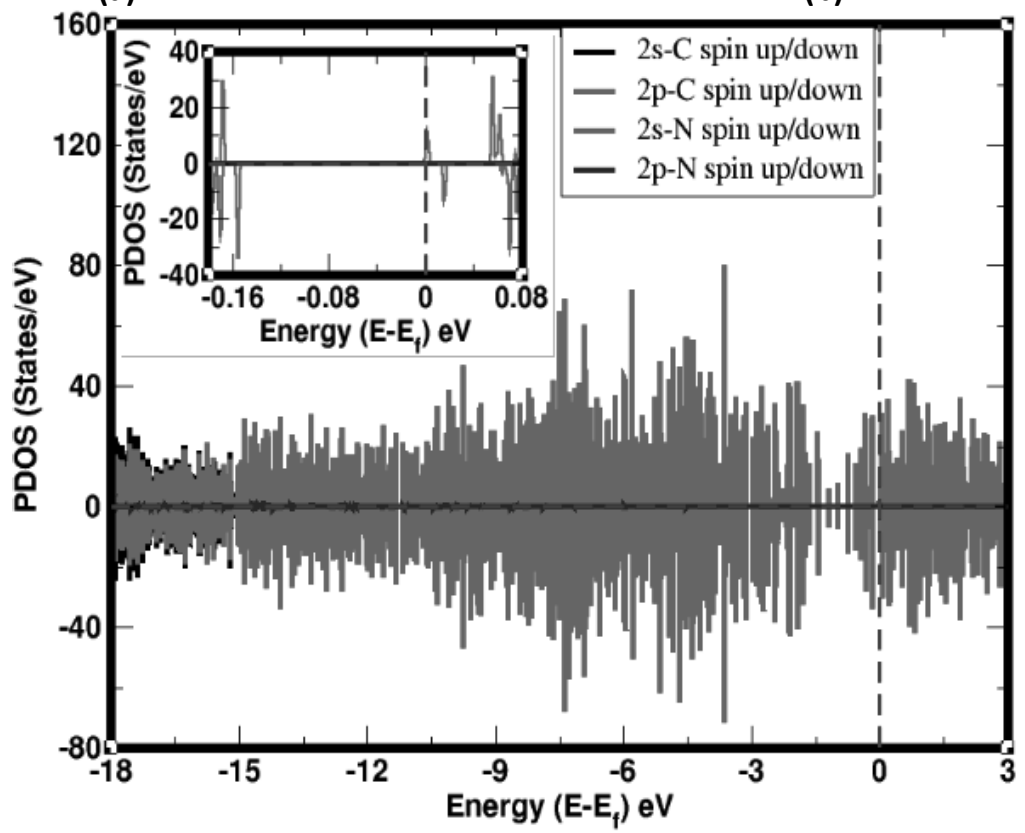

(c)

Fig. 4: (a) Band plot of $1 N$ atom impurity defects in (4×4) supercell structure of graphene, (b) DOS of up \& down spins states of $1 N$ atom impurity defects in $(4 \times 4)$ supercell structure of graphene (c) PDOS of individual up \& down spins states of electrons in the orbitals of $C \& N$ atoms in $1 N$ atom impurity defects in $(4 \times 4)$ supercell structure of graphene. In band structure, horizontal dotted line represents Fermi energy level, and in DOS/PDOS plots, vertical dotted line represents Fermi energy level. 


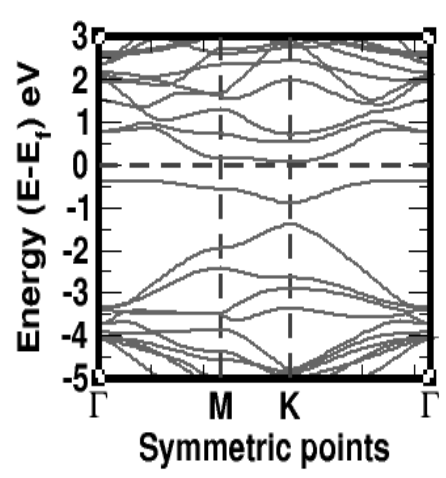

(a)

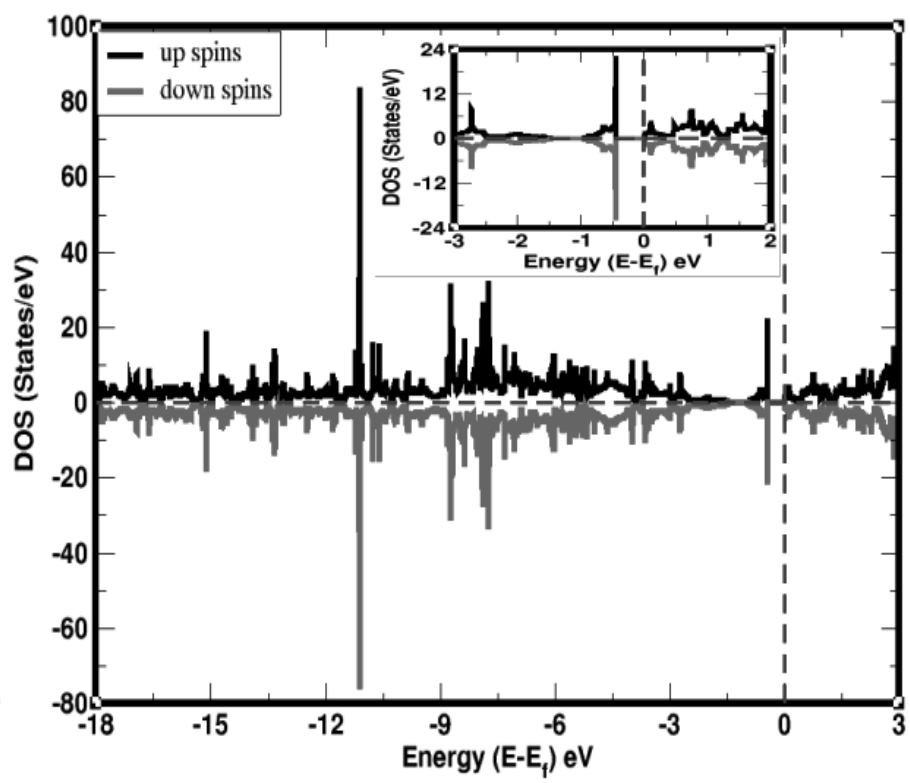

(b)

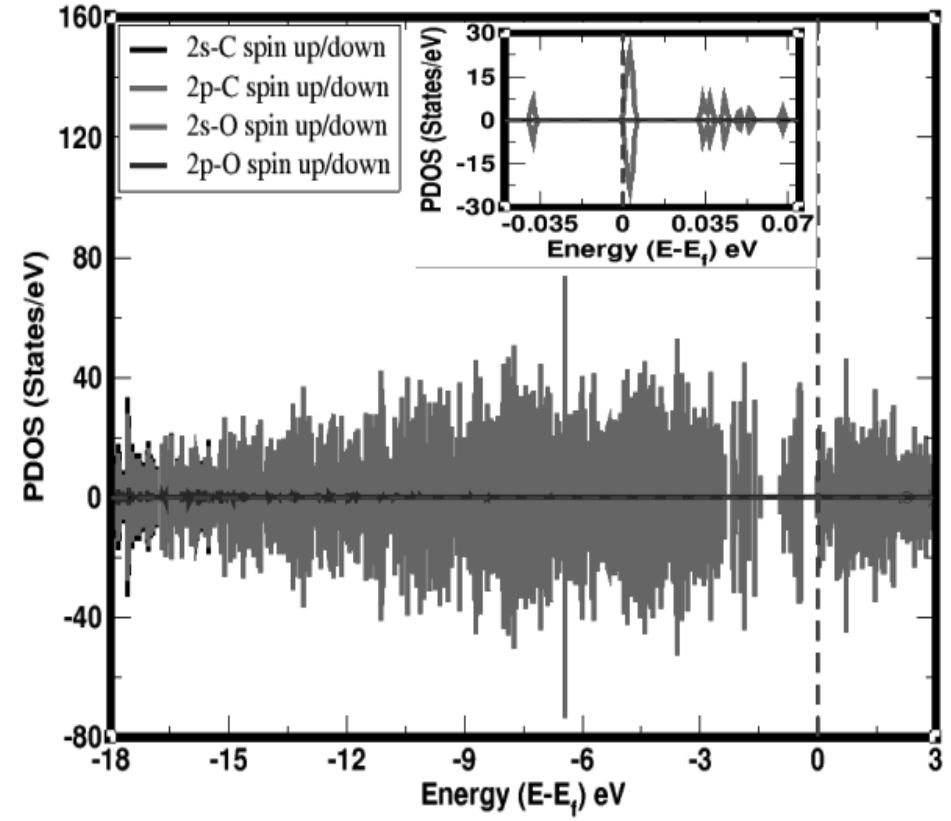

(c)

Fig. 5: (a) Band plot of 10 atom impurity defects in (4×4) supercell structure of graphene, (b) DOS of up \& down spins states of $1 O$ atom impurity defects in $(4 \times 4)$ supercell structure of graphene (c) PDOS of individual up \& down spins states of electrons in the orbitals of $C \& O$ atoms in $1 O$ atom impurity defects in $(4 \times 4)$ supercell structure of graphene. In band structure, horizontal dotted line represents Fermi energy level, and in DOS/PDOS plots, vertical dotted line represents Fermi energy level.

\section{Magnetic properties}

The magnetic properties of materials are studied by DOS and PDOS analysis. DOS suggests how densely quantum states are packed in a particular system. High or zero values of DOS reflects, many or no states are available for occupation. The PDOS calculations are used to investigate the magnetic moment given by distributed up \& down spins states of electrons in the orbital of atoms in a system. The asymmetrically distributed up \& down spins states of electrons in DOS and PDOS means, materials have magnetic properties, and symmetrically distributed up \& down spins states of electrons in DOS and PDOS means, materials carry non-magnetic properties. We observed that up \& down spin states of electrons are asymmetrically distributed near the Fermi energy level in DOS and PDOS plots of $1 \mathrm{C}$ atom vacancy defects supercell structure of graphene $\left(1 \mathrm{C}_{\mathrm{v}-\mathrm{d}}-\mathrm{G}\right)$ as shown in 
figures 3(b-c). This is because electron spins degeneracy of the bands is broken and bands split. Hence, $1 \mathrm{C}_{\mathrm{v}-\mathrm{d}}-\mathrm{G}$ material has magnetic properties. Also, we have calculated the contributions of magnetic moment due to the distribution of spins of electrons in the individual orbital of atoms presented in $1 \mathrm{C}_{\mathrm{v}-\mathrm{d}}-\mathrm{G}$ material as given in table 1 . The magnetic moment developed in material due to up \& down spins of electrons in $2 \mathrm{~s} \& 2 \mathrm{p}$ orbitals of $\mathrm{C}$ atoms have values $-0.22 \mu_{\mathrm{B}} /$ cell $\&-0.53 \mu_{\mathrm{B}} /$ cell respectively. It means, dominant contributions of magnetic moment are given by spins of $2 \mathrm{p}$ orbital of $\mathrm{C}$ atoms in material. Hence, from these calculations, we found that total magnetic moment of $1 \mathrm{C}_{\mathrm{v}-\mathrm{d}}-\mathrm{G}$ material has value $-0.75 \mu_{\mathrm{B}} /$ cell. Therefore, $1 \mathrm{C}_{\mathrm{v}-\mathrm{d}}-\mathrm{G}$ material has magnetic properties.

Similarly, we have calculated the magnetic moment in $1 \mathrm{~N}$ atom impurity defects in graphene $\left(1 \mathrm{~N}_{\mathrm{I}-\mathrm{d}}-\mathrm{G}\right)$ material and $1 \mathrm{O}$ atom impurity defects in graphene $\left(1 \mathrm{O}_{\mathrm{I}-\mathrm{d}}-\mathrm{G}\right)$ material respectively. The DOS and PDOS of up and down spins states of electrons are asymmetrically distributed near the Fermi energy level of $1 \mathrm{~N}_{\mathrm{I}-\mathrm{d}}-\mathrm{G}$ material as shown in figures 4(b-c). Magnetic moment is given by spins states of electrons in the $2 \mathrm{~s}$ and $2 \mathrm{p}$ orbitals of carbon atoms are $0.01 \mu_{\mathrm{B}} /$ cell and $0.03 \mu_{\mathrm{B}} /$ cell respectively, and $2 \mathrm{~s}$ and $2 \mathrm{p}$ orbitals of $\mathrm{N}$ atom are $0.00 \mu_{\mathrm{B}} /$ cell and $0.01 \mu_{\mathrm{B}} /$ cell respectively. Hence, total value of magnetic moment of $1 \mathrm{~N}_{\mathrm{I}-\mathrm{d}}-\mathrm{G}$ material is $0.05 \mu_{\mathrm{B}}$ /cell. Thus, $1 \mathrm{~N}_{\mathrm{I}-\mathrm{d}}$ - $\mathrm{G}$ material has magnetic properties. On the other hand, we have calculated the magnetic moment in 10 atom impurity defects in graphene $\left(1 \mathrm{O}_{\mathrm{I}-\mathrm{d}}-\mathrm{G}\right)$ material. The DOS and PDOS of up and down spins states of electrons are symmetrically distributed near the Fermi energy level of $1 \mathrm{O}_{\mathrm{I}-\mathrm{d}}-\mathrm{G}$ material as shown in figures 5(b-c). Magnetic moment is given by spins states of electrons in the $2 \mathrm{~s}$ and $2 \mathrm{p}$ orbitals of carbon and oxygen atoms are $0.00 \mu_{\mathrm{B}} /$ cell. Thus, total value of magnetic moment of this material has zero value. Therefore, a 10 atom impurity defects in graphene $\left(1 \mathrm{O}_{\mathrm{I}-\mathrm{d}}-\mathrm{G}\right)$ material is a non-magnetic material.

\section{Conclusions}

The electronic and magnetic properties of $1 \mathrm{C}_{\mathrm{v}-\mathrm{d}}-\mathrm{G}, 1 \mathrm{~N}_{\mathrm{I}-\mathrm{d}}-\mathrm{G}$ and $1 \mathrm{O}_{\mathrm{I}-\mathrm{d}}-\mathrm{G}$ materials are investigated by first-principles calculations based on spin-polarized density functional theory. Computational of this work has been done by using Quantum ESPRESSO package. At first, we have prepared (4×4) supercell structure of monolayer graphene sheet, then constructed $1 \mathrm{C}$ atom vacancy defects, and $1 \mathrm{~N}$ atom, $1 \mathrm{O}$ atom respectively impurities defects in monolayer graphene supercell structure. We found that binding energy of defected structures is less than non-defects structure. From the band structure calculations, we found the Dirac cone is formed at the Fermi energy level in graphene material; hence it is called zero bandgap semiconductors. But, electronic band states cross and split around the Fermi energy level in vacancy and impurities defects materials. Hence, defected materials have metallic properties. From the DOS and PDOS calculations, we found that defected $1 \mathrm{C}_{\mathrm{v}-\mathrm{d}}-\mathrm{G}$ and $1 \mathrm{~N}_{\mathrm{I}-\mathrm{d}}-\mathrm{G}$ materials have magnetic properties, but impurity defects $1 \mathrm{O}_{\mathrm{I}-\mathrm{d}}-\mathrm{G}$ material has non-magnetic properties. The non-magnetic nature of graphene supercell structure changes to magnetic materials due to the $1 \mathrm{C}$ atom vacancy defects and $1 \mathrm{~N}$ atom impurity defects in supercell structure of monolayer graphene sheet. The total magnetic moment of $1 \mathrm{C}_{\mathrm{v}-\mathrm{d}}-\mathrm{G}$ and $1 \mathrm{~N}_{\mathrm{I}-\mathrm{d}}-\mathrm{G}$ materials have values $-0.75 \mu_{\mathrm{B}} /$ cell and $0.05 \mu_{\mathrm{B}} /$ cell respectively. The high value of magnetic moment is given by up and down spins states of electrons in $3 p$ orbital of $\mathrm{C}$ atoms in both defected materials.

We can further study about above-mentioned materials by investigating their physical properties using electric field and stress effect.

\section{Acknowledgements}

HKN acknowledges the UGC Nepal Award no. PhD-75/76-S \&T-09. NPA acknowledges network project NT-14 of ICTP/ OEA and UGC Nepal Grants CRG 073/74 -S \& T -01.

\section{References}

1. L. A. Ponomarenko, R. V. Gorbachev, G. L. Yu, D. C. Elias, R. Jalil, A. A. Patel, A.K. Geim. Cloning of Dirac fermions in grapheme superlattices, Nature, 2013, 497(7451), 594-597. (DOI:https://doi.org/10.1038/nature12187).

2. M. Xu, T. Liang, M. Shi, H. Chen. Graphene-like two-dimensional materials, Chemical reviews, 2013, 113(5), 37663798. (DOI: https://doi.org/10.1021/cr300263a). 
3. S. Mayorov, R. V. Gorbachev, S. V. Morozov, L. Britnell, R. Jalil, L. A. Ponomarenko, A. K. Geim. Micrometer-scale ballistic transport in encapsulated graphene at room temperature, Nano letters, 2011, 11(6), 2396-2399. (DOI: https:// doi.org/10.1021/n1200758b).

4. S. V. Morozov, K. S. Novoselov, M. I. Katsnelson, F. Schedin, D. C. Elias, J. A. Jaszczak, A. K. Geim. Giant intrinsic carrier mobilities in graphene and its bilayer, Physical review letters, 2008, 100(1), 016602. (DOI: https://doi.org/10.1103/ PhysRevLett.100.016602).

5. K. S. Novoselov, A. K. Geim, S. V. Morozov, D. Jiang, M. I. Katsnelson, I. Grigorieva, A. A. Firsov. Two-dimensional gas of massless Dirac fermions in grapheme, Nature, 2005,438(7065), 197-200.(DOI:https://doi.org/10.1038/nature04233).

6. J. C. Lei, X. Zhang, Z. Zhou. Recent advances in MXene. Preparation, properties, and applications. Frontiers of Physics, 2015, 10(3), 276-286. (DOI: https://doi.org/10.1007/s11467-015-0493-x).

7. M. E. Dávila, L. Xian, S. Cahangirov, A. Rubio, G. Le Lay Germanene. A novel two-dimensional germanium allotrope akin to graphene and silicene. New Journal of Physics, 2014, 16(9), 095002. (DOI:10.1088/1367-2630/16/9/095002).

8. S. Balendhran, S. Walia, H. Nili, S. Sriram, M. Bhaskaran, Elemental analogues of graphene: silicene, germanene, stanene, and phosphorene, Small, 2015, 11(6), 640-652. (DOI: https://doi.org/10.1002/smll.201402041).

9. C. Kittel, P. McEuen, P. McEuen. Introduction to solid state Physics, New York: Wiley, 1996, pp.140-303. (ISBN: 97881-265-3518-7).

10. H. Lee, Y. W. Son, N. Park, S. Han, J. Yu. Magnetic ordering at the edges of graphitic fragments: Magnetic tail interactions between the edge-localized states, Physical Review B, 2005, 72(17), 174431.(DOI: https://doi.org/10.1103/ PhysRevB.72.174431).

11. S. Okada, A. Oshiyama. Magnetic ordering in hexagonally bonded sheets with first-row elements, Physical review letters, 2001, 87(14), 146803. (DOI:https://doi.org/10.1103/PhysRevLett.87.146803).

12. Y. H. Kim, J. Choi, K. J. Chang, D. Tománek. Defective fullerenes and nanotubes as molecular magnets: an ab initio study, Physical Review B, 2003, 68(12), 125420. (DOI: https://doi.org/10.1103/PhysRevB.68.125420).

13. P. O. Lehtinen, A. S. Foster, Y. Ma, A. V. Krasheninnikov, R. M. Nieminen. Irradiation-induced magnetism in graphite: a density functional study, Physical review letters, 2004, 93(18), 187202. (DOI: https://doi.org/10.1103/ PhysRevLett.93.187202).

14. E. J. Duplock, M. Scheffler, P. J. Lindan, Hallmark of perfect grapheme, Physical review letters, 2004, 92(22), 225502. (DOI: https://doi.org/10.1103/PhysRevLett.92.225502).

15. J. M. Carlsson, M. Scheffler. Structural, electronic, and chemical properties of nanoporous carbon, Physical review letters, 2006, 96(4), 046806. (DOI: https://doi.org/10.1103/PhysRevLett.96.046806).

16. O. V. Yazyev, S. G. Louie. Topological defects in graphene: Dislocations and grain boundaries, Physical Review B, 2010, 81(19), 195420. (DOI: https://doi.org/10.1103/PhysRevB.81.195420).

17. Hashimoto, K. Suenaga, A. Gloter, K. Urita, S. Iijima. Direct evidence for atomic defects in graphene layers, Nature, 2004, 430(7002), 870-873. (DOI:https://doi.org/10.1038/nature02817).

18. V. Krasheninnikov, F. J. N. M. Banhart. Engineering of nanostructured carbon materials with electron or ion beams, Nature materials, 2007, 6(10), 723-733. (DOI:https://doi.org/10.1038/nmat1996).

19. H. K. Neupane, N. P. Adhikari. Structure, electronic and magnetic properties of 2D Graphene-Molybdenum disulphide $\left(\mathrm{G}-\mathrm{MoS}_{2}\right)$ Heterostructure (HS) with vacancy defects at Mo sites, Computational Condensed Matter, 2020, e00489. (DOI:https://doi.org/10.1016/j.cocom.2020.e00489).

20. W. Robertson, B. Montanari, K. He, C. S. Allen, Y. A. Wu, N. M. Harrison, J. H. Warner. Structural reconstruction of the graphene monovacancy, ACS nano, 2013, 7(5), 4495-4502. (DOI:https://doi.org/10.1021/nn401113r). 
21. H. K. Neupane, N. P. Adhikari. Tuning Structural, Electronic, and Magnetic Properties of C Sites Vacancy Defects in Graphene/ $\mathrm{MoS}_{2}$ van der Waals Heterostructure Materials: A First-Principles Study. Advances in Condensed Matter Physics, 2020, 2020. (DOI:https://doi.org/10.1155/2020/8850701).

22. S. Fedorov, Z. I. Popov, D. A. Fedorov, N. S. Eliseeva, M. V. Serjantova, A. A. Kuzubov. DFT investigation of the influence of ordered vacancies on elastic and magnetic properties of graphene and graphene-like SiC and BN structures, Physica status solidi (b), 2012, 249(12), 2549-2552. (DOI:https://doi.org/10.1002/pssb.201200105).

23. R. Faccio, L. Fernández-Werner, H. Pardo, C. Goyenola, O. N. Ventura, Á. W. Mombrú. Electronic and structural distortions in graphene induced By carbon vacancies and boron doping, The Journal of Physical Chemistry C, 2010, 114(44), 18961-18971. (DOI:https://doi.org/10.1021/jp106764h).

24. H. K. Neupane, N. P. Adhikari. First-principles study of structure, electronic, and magnetic properties of C sites vacancy defects in water adsorbed graphene/MoS 2 van der Waals heterostructures, Journal of Molecular Modeling, 2021, 27(3), 1-12. (DOI:https://doi.org/10.1007/s00894-021-04690-8).

25. P. Hohenberg, W. Kohn. Inhomogeneous electron gas. Physical Review, 1964, 136(3B), B864. (DOI: https://doi. org/10.1103/PhysRev.136.B864).

26. P. Giannozzi, S. Baroni, N. Bonini, M. Calandra, R. Car, C. Cavazzoni, A. Dal Corso. QUANTUM ESPRESSO: modular and open-source software Project for quantum simulations of materials, Journal of Physics: Condensed Matter, 2009, 21(39), 395502. (DOI:10.1088/0953-8984/21/39/395502).

27. J. P. Perdew, K. Burke, M. Ernzerhof. Generalized gradient approximation made simple, Physical review letters, 1996, 77(18), 3865. (DOI: https://doi.org/10.1103/PhysRevLett.77.3865).

28. R. M. Martin, Electronic structure: basic theory and Practical methods. Cambridge university press, (2020). (DOI: 10.1017/9781108555586).

29. B. G. Pfrommer, M. Côté, S. G. Louie, M. L. Cohen. Relaxation of Crystals with the quasi-Newton method. Journal of Computational Physics, 1997, 131(1), 233-240. (DOI:https://doi.org/10.1006/jcph.1996.5612).

30. N. Marzari, D. Vanderbilt, A. De Vita, M. C. Payne. Thermal Contraction and disordering of the Al (110) surface. Physical review Letters, 1999, 82(16), 3296. (DOI: https://doi.org/10.1103/PhysRevLett.82.3296).

31. W. Chen, E. J. Santos, W. Zhu, E. Kaxiras, Z. Zhang, Tuning the electronic and chemical properties of monolayer MoS2 adsorbed on transition metal substrates, Nano letters, 2013, 13(2), 509-514. (DOI:https://doi.org/10.1021/nl303909f).

32. W. Xiong, C. Xia, X. Zhao, T. Wang, Y. Jia, Effects of strain and electric field on electronic structures and Schottky barrier in graphene and SnS hybrid heterostructures, Carbon, 2016, 109, 737-746. (DOI:https://doi.org/10.1016/j. carbon.2016.08.082). 